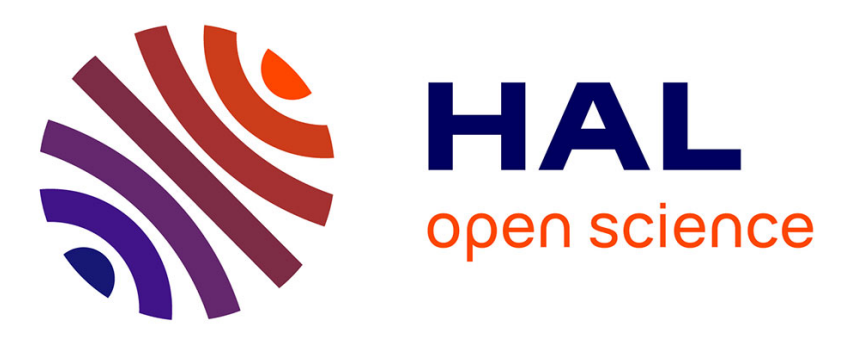

\title{
An agent-based model for mixed traffic in Vietnam based on virtual local lanes
}

Tu Dang-Huu, Benoit Gaudou, Doanh Nguyen-Ngoc, Ngoc Tien Le

\section{To cite this version:}

Tu Dang-Huu, Benoit Gaudou, Doanh Nguyen-Ngoc, Ngoc Tien Le. An agent-based model for mixed traffic in Vietnam based on virtual local lanes. 12th International Conference on Knowledge and Systems Engineering (KSE 2020), Nov 2020, Can Tho city, Vietnam. pp.147-152, 10.1109/KSE50997.2020.9287400 . hal-03086983v1

\section{HAL Id: hal-03086983 \\ https://hal.science/hal-03086983v1}

Submitted on 5 Jan 2021 (v1), last revised 5 Jan 2021 (v2)

HAL is a multi-disciplinary open access archive for the deposit and dissemination of scientific research documents, whether they are published or not. The documents may come from teaching and research institutions in France or abroad, or from public or private research centers.
L'archive ouverte pluridisciplinaire HAL, est destinée au dépôt et à la diffusion de documents scientifiques de niveau recherche, publiés ou non, émanant des établissements d'enseignement et de recherche français ou étrangers, des laboratoires publics ou privés. 


\section{An agent-based model for mixed traffic in Vietnam based on virtual local lanes}

\author{
Tu Dang-Huu \\ Hanoi University of Science and Technology \\ UMMISCO, Sorbonne University, IRD \\ Hanoi, Vietnam \\ tudh.hust@gmail.com \\ Doanh Nguyen-Ngoc \\ Thuyloi University \\ UMMISCO, Sorbonne University, IRD \\ Hanoi, Vietnam \\ doanhnn@tlu.edu.vn
}

\author{
Benoit Gaudou \\ IRIT, Toulouse 1 University Capitole \\ UMMISCO, Sorbonne University, IRD \\ ICTLAb, USTH \\ Toulouse, France \\ benoit.gaudou@gmail.com \\ Ngoc C. Lê \\ Hanoi University of Science and Technology \\ UMMISCO, Sorbonne University, IRD \\ Hanoi, Vietnam \\ ngoc.lechi@hust.edu.vn
}

\begin{abstract}
The rapid urbanization and urban spread in developing countries create a huge number of challenges urban planners have to face. As an example, congestion has become a major issue in Vietnam and caused huge economic losses as well as an increase in air pollutant emissions. Tackling such an issue requires huge improvements in the city organization and infrastructure. Choosing the most appropriate solution among existing ones or imagining new ones require tools to understand their impact and assess their feasibility, effectiveness and acceptability. Computer simulation is a tool of choice to assess the interactions between dynamics at both macro-level (the city) or micro-level (a single road or a crossroad). Existing tools have nevertheless mainly been designed for cities with traffic dominated by cars that are supposed to respect regulations. These assumptions cannot be applied to traffic in Vietnam which is characterized by a mix of cars, motorbikes and other vehicle types and low respect of road lanes. This paper proposes an agent-based model simulating in a more realistic way the traffic in Vietnam using the GAMA platform. We illustrate its capabilities on two characteristic toy case studies (straight road and crossroad) and a real case study in rush hour and lower traffic situations.
\end{abstract}

Index Terms-Agent-Based Model, mixed traffic, traffic simulation, GAMA platform.

\section{INTRODUCTION}

The rapid urbanization in developing countries [1] is the source of a huge number of challenges urban planners have to face, among which traffic and transportation are of first importance. They do not have only a strong impact on the quality of life of city inhabitants, but also have a significant impact on the economy [2] and air pollution. Decision-makers have paid increasing attention to these issues, thinking on how to improve the city organization and infrastructure. Choosing the most appropriate solution among existing ones and even imagining new ones require tools to understand their impact and assess their feasibility, effectiveness and acceptability. Computer simulations are definitely a tool of choice to optimize traffic flow, assess solutions for traffic congestion or infrastructure improvement, and help urban planners to understand interactions between traffic at both macro-level (the city) or micro-level (a single road or a crossroad). Traffic simulations can also be used to test hypothetical scenarios that would be difficult to capture in real life.

Such simulators have been used for years. Nevertheless, existing tools have mainly been designed for cities with traffic dominated by cars, that are expected to respect regulations (e.g. [3], [4] ). These assumptions cannot be applied to traffic in Vietnam because its traffic is characterized by (i) a traffic flow composed of a mixture of many types of transport modes such as bus, car, motorcycle, truck...; (ii) an increase of the vehicle number (due to urban growth) faster than the improvement of the infrastructures; (iii) vehicles which badly comply with traffic rules (traffic lights, or simply the central line of a road).

This paper proposes an agent-based model simulating in a more realistic way the traffic in Vietnam using the GAMA platform [5]: the model is designed to deal with a mixed traffic, without relying on the respect of lanes assumption. We illustrate its capabilities on two characteristic toy case studies (straight road and crossroad) and a real case study (a section of the Giai Phong road in Hanoi) in rush hour and lower traffic situations.

This paper is organized as follows: Section II introduces some state-of-the-art models for traffic simulation. The proposed model is described in Section III. Section IV presents some experiments and results of this model. Finally, Section V concludes the paper and opens a discussion about perspectives.

\section{STATE OF THE ART}

\section{A. Traffic flow modeling}

During the last 70 years, a wide variety of models have been proposed to represent and reproduce the traffic flow. [6] 
categorizes these models in three main approaches based on the modeling scale.

Microscopic models describe individually each vehicle with its own characteristics (speed, location...), its driver's behavior, and its interactions with other vehicles. The traffic flow will emerge from individuals' behaviors and interactions. For instance, [7] first introduced a cellular automaton model for traffic simulation on highway roads and this model was then extended to different traffic networks [3]. Later agentbased models were able to develop more complex models with real geographical data or multi-lanes roads [8]. Microscopic models are highly expressive, as they allow modelers to introduce heterogeneities in terms of vehicle characteristics and drivers' behaviors. However, their computational complexity increases rapidly with the number of vehicles.

On the other hand, macroscopic models describe traffic at a high level of aggregation (in general a road section) by focusing on macroscopic variables such as the vehicle density or the average speed and describing their evolution with equations close to fluid mechanics (e.g. the LWR model [9], [10]). Individual vehicles are thus never represented explicitly. Such models are particularly relevant to simulate traffic in large (city-scale) areas with a huge number of vehicles. Although macroscopic models can generate realistic outputs with low computational costs, they cannot represent different individual vehicles' features or drivers' individual behaviors.

Mesoscopic models are hybrid models combining the expressivity of microscopic models and the computational efficiency of macroscopic models: they can thus describe traffic at a high level of details for large study areas. As an example, the Underwood model [11] considers individual vehicles moving on a road network, but their speed is constrained by the total number of vehicles, computed at the scale of the road section.

\section{B. Traffic in Vietnam}

Given its specificities, several dedicated models have been proposed to represent the traffic in Vietnam (but which could be used more generally to many mixed-traffic situations). As an example, [12] introduced a 2-dimensional model based on the classical Social Force Model [13] (used for example to model crowds) to simulate the traffic at the intersections. [14] also developed a 2-dimensional model, with limited direction choices to reach their target; the model is used to assess various road systems alternatives. [15] developed an agentbased model with agents having the capability to violate regulations. This model has been used to simulate the traffic at the scale of the whole Hanoi city with real data in terms of the road network, population distribution and demographic data (age and job distribution).

\section{MODEL OF TRAFFIC}

In this section, we describe the proposed agent-based model for mixed traffic simulation in Vietnam.

\section{A. Model principles}

The objective of this model is to be able to represent several specific characteristics of the traffic in Vietnam. In particular, it should be able to simulate a mixed traffic, including at least motorbikes, cars and buses. Each type of vehicle will take a specific space on the road and can have a specific maximum speed. An important consequence is that we need to implement a 2-dimensional model, i.e. a model where vehicles can move on all the surface of the road and not only on 1-dimensional lanes: this allows to have 3 motorbikes side by side where only 1 car could be located. Finally, vehicles cannot be considered as respectful of the traffic regulations, such as the middle line splitting the roads into 2 sides or the traffic lights.

Contrarily to pedestrians, vehicles cannot physically move freely in a 2-dimensional space, preventing us to use a Social Force-like model. As a consequence, we propose a model based on virtual local lanes: given a global target (the end of the road section) it wants to reach, each vehicle will choose its next move (the point it will reach during 1 simulation step) among three possible targets located on its front, left and right local virtual lanes (cf. Fig. 1). This choice will depend on the vehicle size, its speed, the other vehicles locations, and traffic regulations (including traffic light and road middle line). This means that if a vehicle is close to the road center, and has slower vehicles in front of it and on its right, it can choose to cross the middle line and continue its way on the wrong side of the road before returning to its lane ${ }^{1}$.

The proposed model includes thus three types of agents: road agents, traffic light agents, and vehicle agents.

\section{B. Road agents}

Road agents represent the basic elements of the road system. Their geometry will be the place where vehicles will move on. Fig. 3 illustrates a crossroad composed of 4 road agents, each of them is a two-way road, and thus contains a middle line, separating its 2 sides (opposite directions). A road agent is thus characterised by 2 attributes: (i) its width, and (ii) Is two-way (true if the road is two-way and false otherwise). Roads are supposed to be static elements in the model and do not have any behavior.

\section{Traffic light agents}

Traffic light agents are used to control the movement of vehicles to organize the traffic in particular in crossroads. Fig. 3 shows an example of such a crossroad between 4 roads with 1 traffic light agent linked to each road.

1) Attributes: A traffic light agent is mainly characterized by its color state attribute (green, yellow or red) and a counter (counting the duration spent in a given color state). In addition, it has 3 additional attributes $t_{g}, t_{y}$, and $t_{r}$ representing the duration of each color state.

2) Behavior: The behavior of a traffic light agent is limited to its color state update. Every simulation step, its counter is incremented. When the counter value is higher than the duration of its current state, it switches to the next state (and reset the counter): after red comes green, after green comes yellow, and after yellow comes red.

\footnotetext{
${ }^{1}$ This mechanism should not be understanding as overtaking a slower vehicle: vehicles can use the whole surface of the road to move and do not plan to come back on the right side when it has to overtake other vehicles.
} 


\section{Vehicle agents}

Vehicle agents represent the various entities moving on the road agents. They can be of various types such as bus, car, motorbike... In this model, we merge vehicles and their drivers in a unique agent.

1) Attributes: Vehicle agents are first of all characterized by their physical attributes: their length (denoted $L$ ), width $(W)$, minimal safe distance to avoid collisions above and behind $(d f)$ and on the sides $(d b)$ as illustrated in Fig. 1. All these values depend on the vehicle type (among car, motorbike and bus).

Vehicles move at a current speed (denoted $v$ ) that is limited by the maximum speed $\left(v_{\max }\right)$ allowed by the traffic regulation. During their trip, vehicles can increase (by an acceleration factor $\alpha$ ) or decrease (by a deceleration factor $\beta$ ) their current speed depending on the other vehicles and traffic laws.

Vehicles are created on a source node of the road network and they will move to reach a final node. The path between these 2 nodes will be represented by a set of road sections. The vehicle start node attribute contains the origin node of the current road on which the vehicle is located, while end node is the target node on the road, that the vehicle wants to reach.

While a vehicle agent has to follow the road direction when moving (toward the end node), its step-by-step move is affected by other vehicles in front of it. To produce traffic based on flexible virtual local lanes, each vehicle computes three rectangular spaces (the virtual local lanes) in front of it (Left, Front, Right attributes in Fig. 1). The location of Front is computed from the vehicle location, the start node and the target node, whereas the size depends on its type of vehicle $(L$ and $W)$. Likewise, Right and Left are on each side of Front and have the same length but half of the Front width. The current vehicle target (i.e. the location that will be targeted in the next move) is computed as the center of one of these three geometries (as detailed in the next section), depending on the location of other vehicles on these geometries (which is stored in the Check go Front / Check go Left / Check go Right attributes). Furthermore, the length of the virtual local lanes (denoted as $l$ ) is dynamic and depends on vehicle speed and type:

$$
l=\left(\phi+\frac{v}{v_{\max }}\right) * L
$$

where $\phi$ is a constant.

Finally, vehicle agents are characterized by a probability to go on the opposite side $(P)$ when possible (e.g. if the road is two-way) and needed. It represents their degree of fulfilment to the regulations.

2) Skills: Vehicle agents have been given the following skills (i.e. the possible actions they are capable to execute; the way and order in which they are called describes the agent's behavior):

- Compute path: finds a list of roads that a vehicle must follow from Source node to Final node, using Dijkstra's algorithm [16].

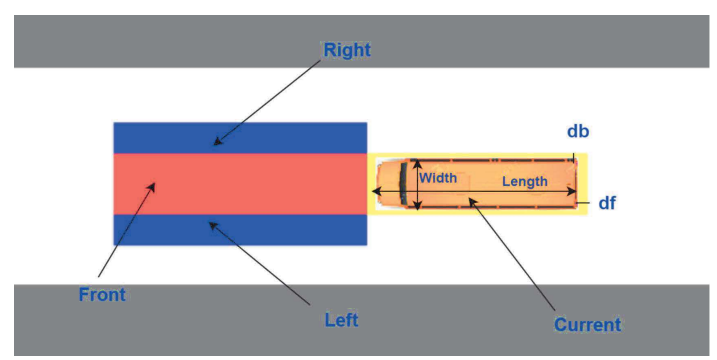

Fig. 1. Example of vehicle attributes.

- Observe obstacles: observes and detects other vehicles in the three rectangular spaces in front of the vehicle.

- Observe traffic lights: observes traffic lights and their state.

- Define new target: chooses the vehicle's new target, depending on surrounding vehicles and traffic lights.

- Move: changes agent location given its current target and speed.

- Accelerate: increases the vehicle speed. The new speed value is computed by this formula:

$$
v=\min \left(v+\alpha, v_{\max }\right)
$$

- Decelerate: decreases the vehicle speed. The new speed value is computed by this formula:

$$
v=\max (v-\beta, 0)
$$

- Stop: reduces the current speed to 0 .

3) Behavior: At the beginning of the first step of the simulation, the vehicle computes its path (i.e. the list of road sections) from its Source node to reach the Final node.

The step-by-step behavior of the vehicle agent is described in the diagram in Fig. 2. After having observed and detected the presence of vehicles in the three Front, Left, Right local virtual lane spaces (and updated the associated attributes Check go Front/Check go Left/Check go Right), it will identify the new target, using the Define new target action.

The choice of the new target depends on the presence of other vehicles, on the basic traffic regulation, and whether the road is one-way or two-way. If it is on an one-way road, the vehicle chooses as a new target the center of (i) Front, if Check go Front is true, (ii) Left, if Check go Left is true, or (iii) Right, if Check go Right is true. The vehicle agent chooses Left before Right when it has the choice as this is a basic traffic recommendation or regulation. If other vehicles are located on the three Front, Left, Right virtual local lanes, the new target is chosen as the central point of Front but the vehicle agent has to decrease its speed to avoid collisions (action Decelerate).

If the road is two-way, the algorithm depends on the location of the vehicle on the road:

- If the vehicle is on its right lane, it checks the location of the Left virtual local lane: if it located on the right side of the road, then the previous algorithm is applied. If Left is located on the wrong side of the road, then the 
vehicle first chooses (i) Front, if Check go Front is true, (ii) Right, if Check go Right is true, or (iii) Left (with the probability $P$ ) or no target (with probability $1-P$ ), if Check go Left is true.

- If the vehicle is on the wrong lane of the two-way road, it chooses (i) Right if Check go Right is true, (ii) Front if Check go Front is true, or (iii) Left (with the probability $P$ ) or no target (with probability $1-P$ ) if Check go Left is true and vehicles in front of it are going in the same direction as it.

- If no target has been chosen, the new target is the central point of Front if the vehicle on the right lane or of Right if the vehicle on the wrong lane. In this case, the vehicle agent has to reduce its speed to avoid collisions (action Decelerate).

When the target has been chosen, if there is no other vehicle in the target space and the vehicle speed has not reached the maximum allowed speed, the vehicle will increase its speed using Accelerate action. Besides that, if a traffic light is detected in the Front geometry, the vehicle will follow the traffic light rules: it will stop if the traffic light is not green and do not change anything if the light is green.

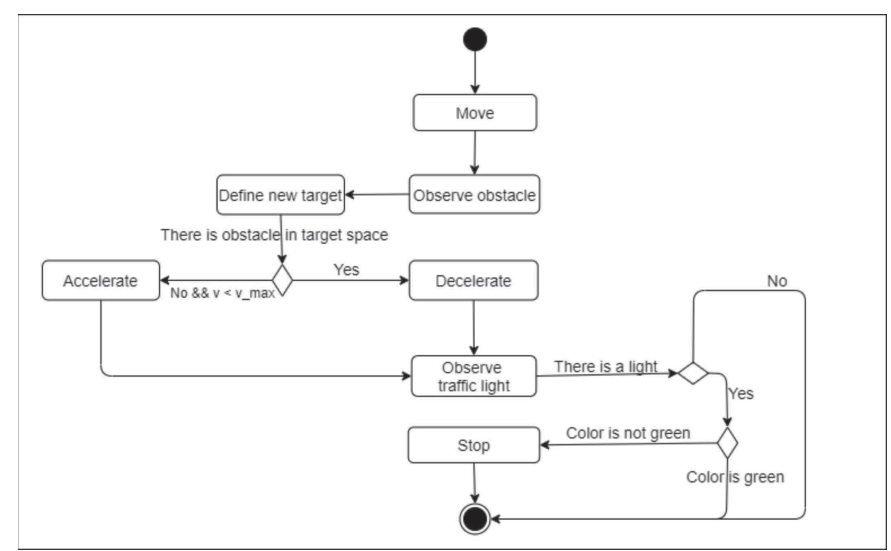

Fig. 2. Activity diagram of a vehicle agent during a simulation step.

\section{EXPERIMENTS AND RESULT}

In this section, we present three applications of the proposed model for traffic simulation in Vietnam. The two first applications illustrate its features on theoretical examples (traffic on a two-way road and in a crossroad). The last application aims at reproducing the traffic on a real situation: a section of the Giai Phong road (Hanoi) during 2 time periods in the morning. The models have been implemented on the GAMA platform [5]. Characteristics of the various kinds of vehicle (bus $^{2}, \mathrm{car}^{3}$, and motorbike ${ }^{4}$ ) are summarized in Table I.

\footnotetext{
${ }^{2}$ Source: https://shac.vn/kich-thuoc-xe-buyt-cac-loai

${ }^{3}$ Source: https://www.carmudi.vn/blog-xe-hoi/kich-thuoc-xe-oto

${ }^{4}$ Source: https://thoisu.com.vn/kich-thuoc-xe-may
}

TABLE I

VALUE OF ATTRIBUTES OF VEHICLES

\begin{tabular}{|c|c|c|c|}
\hline & Bus $^{2}$ & Car $^{3}$ & Motorbike $^{4}$ \\
\hline Length & $9.45 \mathrm{~m}$ & $3.8 \mathrm{~m}$ & $1.9 \mathrm{~m}$ \\
Width & $2.45 \mathrm{~m}$ & $1.5 \mathrm{~m}$ & $0.7 \mathrm{~m}$ \\
$V_{\max }$ & $60 \mathrm{~km} / \mathrm{h}$ & $50 \mathrm{~km} / \mathrm{h}$ & $40 \mathrm{~km} / \mathrm{h}$ \\
df & $0.4 \mathrm{~m}$ & $0.15 \mathrm{~m}$ & $0.1 \mathrm{~m}$ \\
$\mathrm{db}$ & $0.2 \mathrm{~m}$ & $0.1 \mathrm{~m}$ & $0.05 \mathrm{~m}$ \\
\hline
\end{tabular}

\section{A. Simulation of a crossroad}

Traffic simulation at a crossroad is always a difficult problem because there is a large number of vehicles, vehicles may have to cross a flow of vehicles going in a different direction, and the drivers behavior is more complex. In this simulation, the crossroad is designed as an intersection between two twoway roads. Each road is $16 \mathrm{~m}$ wide and the crossroad is controlled by four traffic lights. The duration of each traffic light state is set to: $t_{g}=20 \mathrm{~s}, t_{y}=4 \mathrm{~s}$, and $t_{r}=24 \mathrm{~s}$.

To assess the behavior of our model in such a situation, we run several simulations with different values of vehicle inflow. Simulations show that the vehicles can move easily through the crossroad and there is no congestion with the traffic flow of 2 to 4 vehicles/s (Fig. 3a illustrates only the case of 4 vehicles/s). However, when we increase the traffic flow to 5 vehicles/s, traffic congestion occurs and even a deadlock appears (Fig. 3b). The reason is that with this level of traffic volume, the skills of vehicle agents are not enough sophisticated to handle all conflicts in the crossroad while the number of vehicles at the junction increases rapidly.

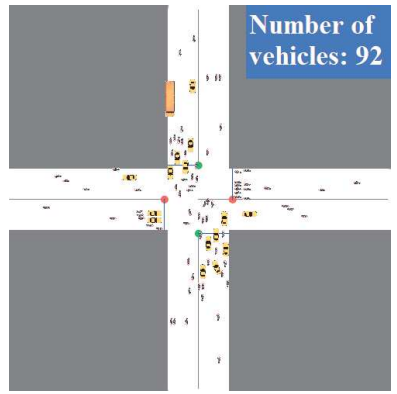

(a) 4 vehicles/s

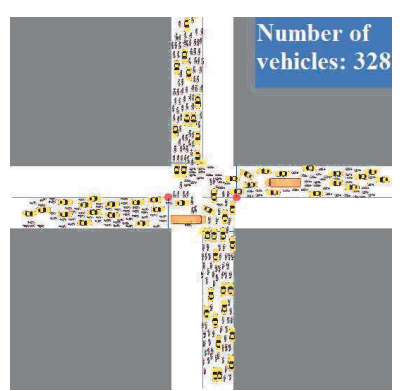

(b) 5 vehicles/s
Fig. 3. Traffic simulations at the crossroad with different inflows.

\section{B. Simulation on two-way roads}

Most two-way roads in Vietnam have only a single solid line to distinguish two opposite directions, requires traffic participants to stay within the lane. But Vietnamese drivers rarely follow these traffic rules if they have a chance to move to the other side to overtake its front vehicle. In particular, depending on the flows in the two entry nodes of the road, the boundary between vehicles going in opposite directions can be different (and not localized on the central line).

To illustrate this feature, we simulate vehicles moving on a straight two-way road which contains only a solid line to 
separate the two directions. The two-way road is 16 meters wide and 200 meters long. We choose $P=1$ : this means that drivers will always take the Left virtual local lane on the opposite road side when Front and Right are occupied by other vehicles. We run 3 experiments with different flows. In Fig. 4a, the traffic volume on both lanes are equal to 5 vehicles/s. In this case, very few vehicles move to the opposite side of the road because each side does not have enough space for the opposite vehicles. We can also observe the red line showing the boundary between the 2 flows is close to the central line and is on both sides alternatively. Fig. $4 \mathrm{~b}$ represents a situation with a slight difference between the upper ( 5 vehicles/s) and lower (3 vehicles/s) lanes inflows. The result shows that traffic in the 2 lanes are not balanced anymore: 5 vehicles from the upper lane move to the lower lane while only 1 vehicle from the lower lane goes to the upper lane. Finally Fig. 4c illustrates the case of a major difference in terms of traffic flows between the upper (5 vehicles/s) and lower (1 vehicle/s) lanes. We can observe clearly in this situation that the upper side is wider than the lower side, due to the higher number of vehicles.

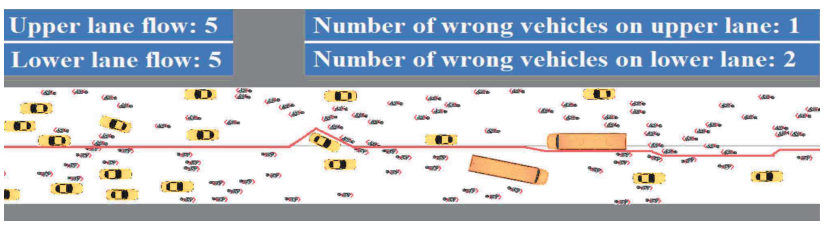

(a)

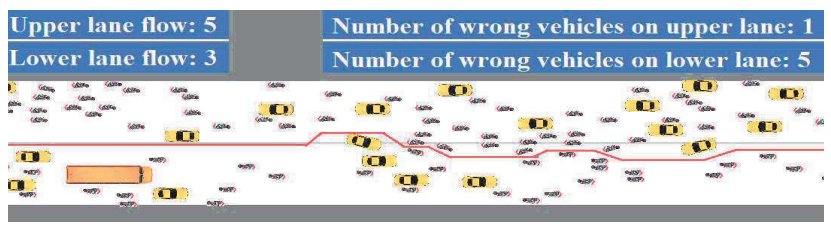

(b)

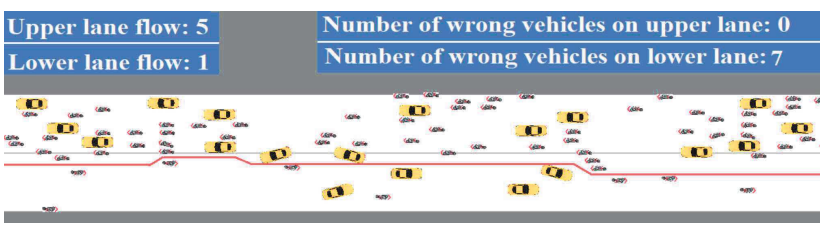

(c)

Fig. 4. Simulation on two-way road with traffic flows for upper and lower lanes: (a) 5 vehicles/s and 5 vehicles/s, (b) 5 vehicles/s and 3 vehicles/s, (c) 5 vehicles/s and 1 vehicle/s.

\section{Study case on Giai Phong road}

1) Problem description: Giai Phong road is one of the most important roads in Hanoi city. The road is two-way, but the two directions are separated by a central island. We choose a segment of one side of Giai Phong road to simulate the traffic. As vehicles cannot travel in the opposite direction, we consider it as a one-way road segment. The selected road section is 200 meters long from Nga Tu Vong intersection to Bach Mai hospital and 10.5 meters wide (see Fig. 5). Our objective is to reproduce the actual traffic in 2 different traffic conditions: rush hour (at 7:30 $\mathrm{AM}$ ) and a sparse traffic period (10:30 $\mathrm{AM})$.

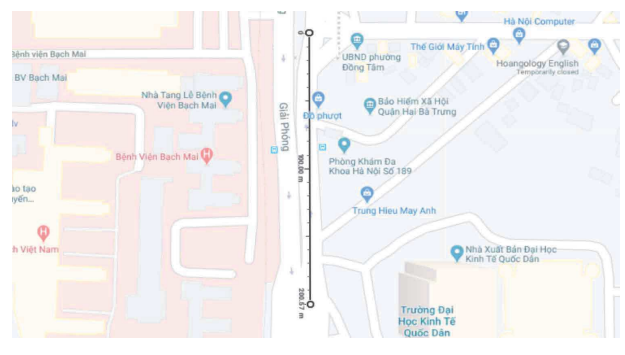

Fig. 5. Selected Giai Phong road segment.

2) Data collection: The traffic data come from a video footage of traffic flow recorded at two different times: 7:30 $\mathrm{AM}$ and 10:30 AM (Fig. 6). We have counted a vehicle flow of 7 vehicles/s at 7:30 AM and 2.5 vehicles/s at 10:30 AM. In addition, the average number of vehicles currently on the road at any given time (Table II) is calculated as the average of the number of vehicles on the road at several time points.

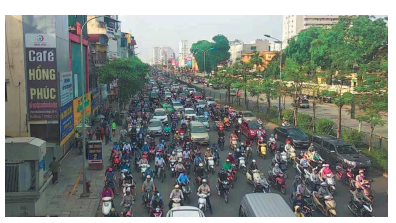

(a)

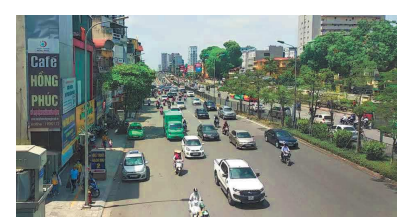

(b)
Fig. 6. Traffic flow on Giai Phong road. (a) 7:30 AM. (b) 10:30 AM.

TABLE II

NUMBER OF EACH TYPE OF VEHICLES.

\begin{tabular}{|c|c|c|c|}
\hline & Bus & Car & Motorbike \\
\hline 7:30 AM & 1 & 38 & 240 \\
\hline 10:30 AM & 1 & 15 & 36 \\
\hline
\end{tabular}

3) Model calibration: Studies [14] show that most of the drivers limit their speed to a safe speed instead of trying to reach the maximum allowed speed. Safe speed depends on the vehicle type and the driver gender (data presented in Table III). In the simulation, we initialize vehicle maximum speed either with the safe speed or the maximum allowed speed with an equal probability. Combined with the traffic volume, we perform traffic simulation for Giai Phong road segment.

TABLE III

SAFE SPEED FOR DIFFERENT VEHICLES AND GENDERS (FROM [14])

\begin{tabular}{|c|c|c|}
\hline & Car & Motorbike \\
\hline Male & $40 \mathrm{~km} / \mathrm{h}$ & $32 \mathrm{~km} / \mathrm{h}$ \\
Female & $36 \mathrm{~km} / \mathrm{h}$ & $30 \mathrm{~km} / \mathrm{h}$ \\
\hline
\end{tabular}

4) Simulation Result: The simulation results focus on two aspects: the average speed of the traffic flow and the average number of vehicles currently on the road segment. 
At 7:30 AM, we set the traffic inflow to 7 vehicles/s. The traffic simulation can be seen in Fig. 7a. In this simulation, the average speed of the vehicles is about $15 \mathrm{~km} / \mathrm{h}$ and the average number of vehicles currently on the road segment is between 300 to 350 vehicles (Fig. 7b). This number is comparable but higher than the measured one (cf. Table II): given the high density, it appears necessary to investigate more precisely the conditions at the beginning and the end of the road sections if we want to better fit to the actual data.

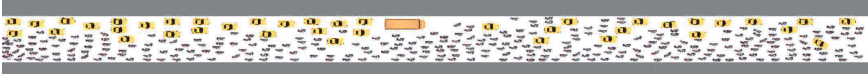

(a)

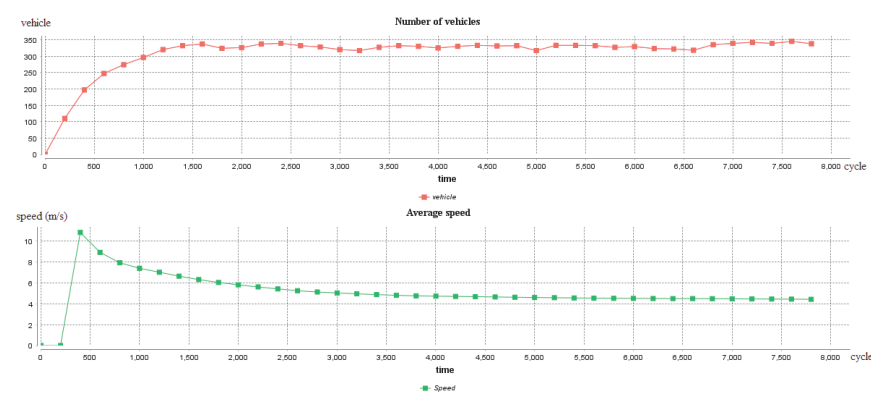

(b)

Fig. 7. (a) Traffic simulation at 7:30 AM. (b) Simulation result at 7:30 AM.

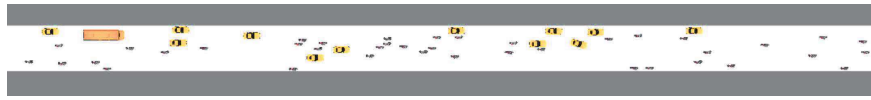

(a)

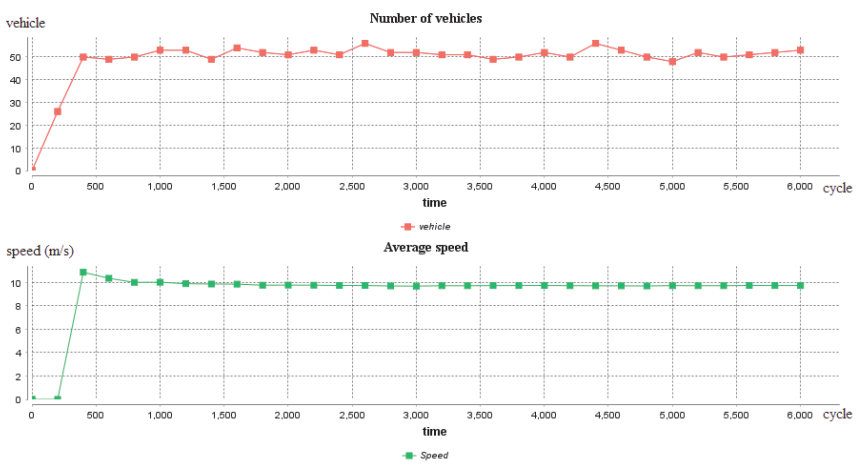

(b)

Fig. 8. (a) Traffic simulation at 10:30 AM. (b) Simulation result at 10:30 AM.

The Fig. 8a shows the traffic at 10:30 AM with a traffic inflow of 2.5 vehicles/s. Simulation results in Fig. 8b shows that the average speed of traffic flow is around $34 \mathrm{~km} / \mathrm{h}$ and the average number of vehicles currently on the road is between 50 and 60 vehicles which are very close to the average number of vehicles at 10:30 AM in Table II. At that time, vehicles can move easily on the road due to sparse traffic at 10:30 AM.

\section{CONCLUSION}

In this paper, we proposed a traffic simulation model based on virtual local lanes for mixed traffic in Vietnam. The model has been applied to simulate traffic on a two-way road and a crossroad to illustrate its capabilities. Results show the model capability to reproduce a common regulation violation behavior observed in Vietnamese traffic: crossing the central line to move on the opposite side of the road, which is a novelty compared to existing models of mixed traffic in Vietnam. In addition, our model has shown its capability to reproduce an observed traffic jam situation (Giai Phong road, Hanoi, at 7:30 AM). In order to go one step further, and use it in a decision-support tool, it is nevertheless necessary to improve the model to be apply it on a real road network created from actual GIS data. In addition, the improvement of individual behaviors and comparison with existing simulation methods need to be investigated to better understand and forecast complex issues such as crossroad deadlocks.

\section{ACKNOWLEDGMENT}

The group received the support from Vietnam Institute for Advanced Study in Mathematics, Year 2020.

\section{REFERENCES}

[1] U. D. of Economic and S. Affairs, "World urbanization prospects - the 2018 revision," United Nation, Tech. Rep., 2019. [Online]. Available: https://population.un.org/wup/Publications/Files/WUP2018-Report.pdf

[2] N. Minh Ha, L. Nguyen, and Trung-Kien, "The impact of urbanization on income inequality: A study in vietnam," Journal of Risk and Financial Management, vol. 12, p. 146, 2019.

[3] A. Schadschneider and M. Schreckenberg, "Cellular automation models and traffic flow," J. of Phys. A: Math. and Gen., vol. 26, no. 15, 1993

[4] M. Rickert, K. Nagel, M. Schreckenberg, and A. Latour, "Two lane traffic simulations using cellular automata," Physica A: Statistical Mechanics and its Applications, vol. 231, no. 4, pp. 534-550, 1996.

[5] P. Taillandier, B. Gaudou, A. Grignard, Q.-N. Huynh, N. Marilleau, P. Caillou, D. Philippon, and A. Drogoul, "Building, composing and experimenting complex spatial models with the GAMA platform," GeoInformatica, vol. 23, no. 2, pp. 299-322, 2019.

[6] S. P. Hoogendoorn and P. H. L. Bovy, "State-of-the-art of vehicular traffic flow modelling," Journal of Systems and Control Engineering, vol. 215, no. 4, pp. 283-303, 2001.

[7] K. Nagel and M. Schreckenberg, "A cellular automaton model for freeway traffic," J. de physique I, vol. 2, no. 12, pp. 2221-2229, 1992.

[8] P. Taillandier, "Traffic simulation with the gama platform," in Eighth Int. Workshop on Agents in Traffic and Transportation, 2014, pp. 8-p.

[9] M. J. Lighthill and G. B. Whitham, "On kinematic waves II. A theory of traffic flow on long crowded roads," Proc. of the Royal Soc. of Lond. Series A. Math. and Phys. Sc., vol. 229, no. 1178, pp. 317-345, 1955.

[10] P. I. Richards, "Shock waves on the highway," Operations research, vol. 4 , no. 1 , pp. $42-51,1956$.

[11] R. T. Underwood, "Speed, volume and density relationships," Quality and theory of traffic flow, 1961.

[12] D. N. Huynh, M. Boltze, and A. T. VU, "Modelling mixed traffic flow at signalized intersectionusing social force model," Journal of the Eastern Asia Society for Transportation Studies, vol. 10, pp. 1734-1749, 2013.

[13] D. Helbing and P. Molnar, "Social force model for pedestrian dynamics," Physical Review E, vol. 51, 1998.

[14] T. D. Bui, D. H. Ngo, and C. Tran, "Multi-agent based simulation of traffic in vietnam," in Principles and Practice of Multi-Agent Systems. PRIMA 2010, vol. 7057. Springer, 2010, pp. 636-648.

[15] M. H. Nguyen and T. V. Ho, "An agent-based model for simulation of traffic network status: Applied to hanoi city," Simulation, vol. 92, no. 11, pp. 999-1012, 2016.

[16] D. E. Knuth, "A generalization of Dijkstra's algorithm," Information Processing Letters, vol. 6, no. 1, pp. 1-5, 1977. 